\title{
The Validity and Reliability of a New Modified Persian Version of DN4 to Discriminate Non- neuropathic from Neuropathic
}

\author{
Seyed Pezhman Madani', Korosh Mansouri', Shahla Asadi ${ }^{*}$, Safoora Ebadi ${ }^{1}$ \\ ${ }^{1}$ Neuromusculoskletal Research Center, Department of Physical Medicine and Rehabilitation, Iran University of \\ Medical Sciences, Tehran, Iran
}

*Correspondence to

Shahla Asadi,

Email: shahlaasadi666@gmail.com

Received October 15, 2020 Accepted January 15, 2021 Published online March 31, 2021

\begin{abstract}
Introduction: One of the popular questionnaires for screening neuropathic pain is the DN4 (Douleur Neuropathique 4) which has been translated into many languages and validated in different populations. So, we wanted to determine the Persian version of this questionnaire's reliability and validity to differentiate neuropathic pain from other painful non-neuropathic conditions.

Methods: This cross-sectional study was conducted on 120 patients who suffered from chronic pain referred to the Physical Medicine and Rehabilitation Department at the three great referral hospitals in Tehran, Iran in 2016. In this study, a modified version of DN4 was used to validate adding a question related to the appearance of pain in an especial joint and adding visual analogue scaling (VAS) score in each item.

Results: The modified DN4 questionnaire had high reliability for assessing neuropathic pain with the Cronbach's alpha coefficient of 0.874 . The correlation coefficients obtained by the retest intra-class correlation test ranged from 0.650 to 0.941 . Also, the internal consistency of Spearman's R coefficients ranged from 0.482 to 0.904 . Test-retest reliability analysis also showed a higher intraclass correlation (ICC) value in the neuropathic than non-neuropathic pain group (0.85 vs0.73). The modified DN4 questionnaire could effectively discriminate neuropathic pain from non-neuropathic pain with the area under the curve (ROC) of 0.989 (95\% Cl: 971-1000, $P<0.001)$. The best cutoff value for the modified DN4 questionnaire to diagnose neuropathic pain was 5 , yielding a sensitivity of $98.3 \%$ and a specificity of $90.3 \%$.

Conclusion: Applying the new modified Persian version of DN4 achieved by adding a VAS score and a new question related to the appearance of pain in an especial joint led to high reliability and validity for this tool to discriminate neuropathic from non-neuropathic pain.

Keywords: DN4, Neuropathic pain, Persian version, Questionnaire, Reliability, Validity
\end{abstract}

\section{Introduction}

Neuropathic pain is a complex problematic condition characterized by chronic pain accompanied by injury and dysfunction of the nerve fibers especially the somatosensory system. ${ }^{1}$ Etiologically, several causes including diabetes mellitus, alcoholism, musculoskeletal disorders, nerve injuries, multiple sclerosis, parkinsonism, chemotherapy-induced neuropathy, tumor infiltration, spinal surgical interventions, shingles, HIV infection or post-herpetic neuralgia have been suggested. ${ }^{2-4}$ Neuropathic pain is usually described as a burning or shooting pain with numbness and tingling. In this regard, this pain diagnosis is based on interviews and accurate physical examination to discover the primary source of pain and its-related triggering. ${ }^{5}$ Unfortunately, no gold standard tool has been introduced for the final diagnosis of neuropathic pain. However, a combination of physical assessment and neurological tests is employed to achieve the final diagnosis. ${ }^{6}$ According to "The European Federation of Neurological Sciences guidelines", describing disease symptoms by the patients, along with stimulus- evoked pains induced by a mechanical, chemical, or thermal stimulus, can be potentially diagnostic. ${ }^{7}$ Besides diagnostic tools, some

(C) 2021 The Author(s); Published by Zabol University of Medical Sciences. This is an open-access article distributed under the terms of the Creative Commons Attribution License (http://creativecommons.org/licenses/by/4.0), which permits unrestricted use, distribution, and reproduction in any medium, provided the original work is properly cited. 
screening instruments and questionnaires have been developed to screen the neuropathic pain and differentiate it from other painful conditions such as nociceptive pain. One of the most used questionnaires for screening neuropathic pain is the DN4 (Douleur Neuropathique 4) which was primarily structured in French version. ${ }^{8}$ This tool has some items about how the patient feels the pain. Also, it has items requiring professional assessment of reduced or increased sensation (hypoaesthesia vs allodynia). ${ }^{9}$ The DN4 has been translated into different languages and validated in diverse populations. The present study aimed to determine the modified Persian version of this questionnaire's reliability and validity to identify neuropathic pain from other painful nonneuropathic conditions.

\section{Materials and Methods \\ Study Population}

This cross-sectional study was conducted on 120 consecutive patients who suffered from chronic pain referred to the Physical Medicine and Rehabilitation Department at three great referral hospitals in Tehran (Firoozgar hospital, Shafa yahyaeian hospital, Rasul Akram hospital), Iran, in 2016.

The eligible subjects for entering the study include patients aged higher than 18 years suffering chronic pain within the last three months, regardless of its neuropathic or non-neuropathic nature. The severity of pain was initially determined according to the visual analogue scaling (VAS) method ranged from 0 to 110 . Exclusion criteria were any syndromes with unknown origin, pain induced by malignancies, complex regional pain syndrome, headache, visceral pains, severe depression, history of using alcohol or substances abuse.

The primary diagnosis was based on the interview and physical examination done by a physical medicine expert. Two physicians physically examined the patients at two times within 24 to 48 hours and fulfilled the questionnaires. Ethically, the Declaration of Helsinki principles were respected. Entering the study was subject to the prior written consent of the patients. The principal researcher had adequately explained the objectives and methodology, and informed consent was signed before recruiting the patient.

\section{Tool Description}

In this study, a modified version of DN4 was used to validate adding a question and adding a VAS score in each question. The questionnaire's first three items were related to the quality of pain, including tingling, painful cold or electric shock sensation. The other four questions described abnormal feelings of pain, such as tingling, numbness, and itching

Three items of the questionnaire were physical assessment and had been performed by the physician. The added question in our tool was about pain in an especial joint. For each item, "1" was the score for "yes" and " 0 " for "no." The patient is defined to have neuropathic pain by a total score of 4 or more.

\section{The Method of Validation}

To assess the modified DN4 questionnaire's reliability and internal consistency, reliability analysis (by calculating the Cronbach's alpha coefficient) and the Spearman correlation test (by calculating correlation coefficient) were used.

The Kappa value was also determined to assess agreement between the two experts who completed the questionnaires.

\section{Statistical Analysis}

Statistical analyses were performed in SPSS 22 (SPSS Inc., Chicago, IL). Mean \pm standard deviation (SD) was used to describe quantitative data, and percentages and frequencies were applied for categorical variables. The Kolmogorov-Smirnov test was used to assess normality. The statistical tests of chi-square test or Fisher exact test were used to investigate the relationship between categorical variables. Also, the student $t$ test (for normally distributed) or Mann-Whitney U test (for non-normally distributed) were used to compare means between groups. The correlation between the study parameters was assessed using the Pearson's correlation test. The area under the curve (ROC), and the best cutoff point for the DN4 total score to achieve optimal sensitivity and specificity for diagnosing neuropathic pain were also determined.

For the statistical analysis, the statistical software SPSS version 22.0 for windows (SPSS Inc., Chicago, IL) was used. P values of 0.05 or less were considered statistically significant.

\section{Results}

In total, 120 patients (78 women and 42 men) were entered. Neuropathic pain was diagnosed in 61 patients (41 women and 20 men), and thus 59 patients ( 37 women and 22 men) suffered from non-neuropathic pain with no difference between the two genders $(P=0.7)$. The average age of participants was $49.99 \pm 16.27$ years (ranged from 20 to 89 years). No difference was revealed in mean age between the patients with neuropathic (50.96 \pm 15.92 years) and non-

neuropathic pain $(48.98 \pm 16.71$ years $) \quad(P=0.7)$. Regarding occupational status, in total, 75 patients $(62.5 \%)$ were employed that the rate of employment in neuropathic pain and non-neuropathic pain groups was $65.6 \%$ and $59.4 \%$, respectively $(P=0.57)$ Regarding educational level, an academic degree was revealed in $32.8 \%$ of patients with neuropathic pain and $18.6 \%$ of those with non-neuropathic pain with no difference 
$(P=0.09) .65 .6 \%$ of patients with neuropathic pain use analgesic drugs, which is significantly higher than those who suffered non-neuropathic pain (40.7\%) $(P=0.01)$. The mean body mass index $(\mathrm{BMI})$ in the neuropathic pain group was $19.40 \pm 38.10 \mathrm{~kg} / \mathrm{m}^{2}$, and in the non-neuropathic pain group was $16.20 \pm 36.20 \mathrm{~kg} / \mathrm{m}^{2}$ $(P<0.01)$. Regarding the severity of pain based on the VAS scoring system, the mean VAS score in the neuropathic pain group was $73.81 \pm 4.63$ and in the non-neuropathic pain group $68.68 \pm 3.61$ revealing more severe pain in the former group $(P<0.001)$. The mean DN4 score for patients with neuropathic pain was $7.11 \pm 1.30$ that was significantly higher when compared to those with nonneuropathic pain $(1.02 \pm 1.01)(P<0.001)$. Also, the mean score reported by the two interviewers was 3.97 and 4.11 , indicating no difference.

As shown in Table 1, the modified DN4 questionnaire had high reliability for assessing neuropathic pain with the Cronbach's alpha coefficient of 0.874 ). The correlation coefficients obtained by the retest intra-class correlation test ranged from 0.650 to 0.941 . Also, Spearman's $r$ coefficients ranged from 0.482 to 0.904 indicating high internal consistency reliability for the questionnaire.

The kappa agreement test showed a high agreement value between the two questioners (kappa value of 0.520). Test-retest reliability analysis (Table 2) also showed higher intraclass correlation (ICC) value in the neuropathic pain group (0.85) than in the non-neuropathic pain group (0.73).

Using the ROC curve analysis, the DN4 system could effectively discriminate neuropathic pain group from non-neuropathic pain with the area under the ROC of 0.989 (95\% CI: 971-1000, $P<0.001$ ) (Figure 1). The diagnostic performance of different cutoff points for DN4 is described in Table 3.

The best cutoff value for DN4 to diagnose neuropathic pain was 5 yielding a sensitivity of $98.3 \%$ and a specificity of $90.3 \%$. In a sub-analysis based on the pain severity, the value of DN4 for predicting neuropathic pain was significantly higher in those with VAS $\geq 70$ than in patients

Table 1. Reliability of DN4 by Considering Each Item of the Tool

\begin{tabular}{|c|c|c|c|c|}
\hline Items & $\begin{array}{l}\text { Internal-Rater Agreement } \\
\text { Kappa Cohen's }\end{array}$ & Test-Retest ICC & $\begin{array}{c}\text { Reliability Cronbach Alpha } \\
\text { Coefficient }\end{array}$ & $\begin{array}{c}\text { Internal Consistency } \\
\text { Spearman's r Coefficient }\end{array}$ \\
\hline Burning & $\begin{array}{l}0.833 \\
\text { SEM: } 0.050\end{array}$ & 0.879 & \multirow{3}{*}{0.455} & 0.783 \\
\hline Painful cold & $\begin{array}{l}0.777 \\
\text { SEM: } 0.064\end{array}$ & 0.851 & & 0.742 \\
\hline Electric shocks & $\begin{array}{c}0.520 \\
\text { SEM: } 0.105\end{array}$ & 0.650 & & 0.482 \\
\hline Tingling & $\begin{array}{l}0.850 \\
\text { SEM: } 0.048\end{array}$ & 0.920 & \multirow{4}{*}{0.693} & 0.851 \\
\hline Pins and needles & $\begin{array}{c}0.883 \\
\text { SEM: } 0.043\end{array}$ & 0.941 & & 0.889 \\
\hline Numbness & $\begin{array}{c}0.881 \\
\text { SEM: } 0.044\end{array}$ & 0.938 & & 0.884 \\
\hline Itching & $\begin{array}{c}0.635 \\
\text { SEM: } 0.100\end{array}$ & 0.783 & & 0.652 \\
\hline Hypoesthesia to touch & $\begin{array}{l}0.850 \\
\text { SEM: } 0.048\end{array}$ & 0.921 & \multirow{2}{*}{0.808} & 0.853 \\
\hline Hypoesthesia to prick & $\begin{array}{c}0.882 \\
\text { SEM: } 0.043\end{array}$ & 0.938 & & 0.884 \\
\hline Brushing & $\begin{array}{c}0.769 \\
\text { SEM: } 0.078\end{array}$ & 0.869 & - & 0.769 \\
\hline Joint pain & $\begin{array}{l}0.899 \\
\text { SEM: } 0.040\end{array}$ & 0.949 & - & 0.904 \\
\hline Total & & 0.979 & 0.874 & \\
\hline
\end{tabular}

Table 2. Test-Retest Reliability in Both Neuropathic and Non-neuropathic Pain Groups

\begin{tabular}{|c|c|c|c|}
\hline & \multicolumn{3}{|c|}{ Test-Retest Reliability } \\
\hline & $\begin{array}{c}\text { First Assessment } \\
\text { Mean (SD) }\end{array}$ & $\begin{array}{c}\text { Second Assessment } \\
\text { Mean (SD) }\end{array}$ & $\begin{array}{c}\text { ICC } \\
(95 \% \mathrm{CI})\end{array}$ \\
\hline Total & $3.97(3.25)$ & $4.11(3.27)$ & $0.975(0.96-0.98)$ \\
\hline Neuropathic pain group & $6.86(1.37)$ & $7.11(1.31)$ & $0.85(0.75-0.91)$ \\
\hline Non-neuropathic pain group & $0.97(1.32)$ & $1.01(1.02)$ & $0.73(0.54-0.84)$ \\
\hline
\end{tabular}




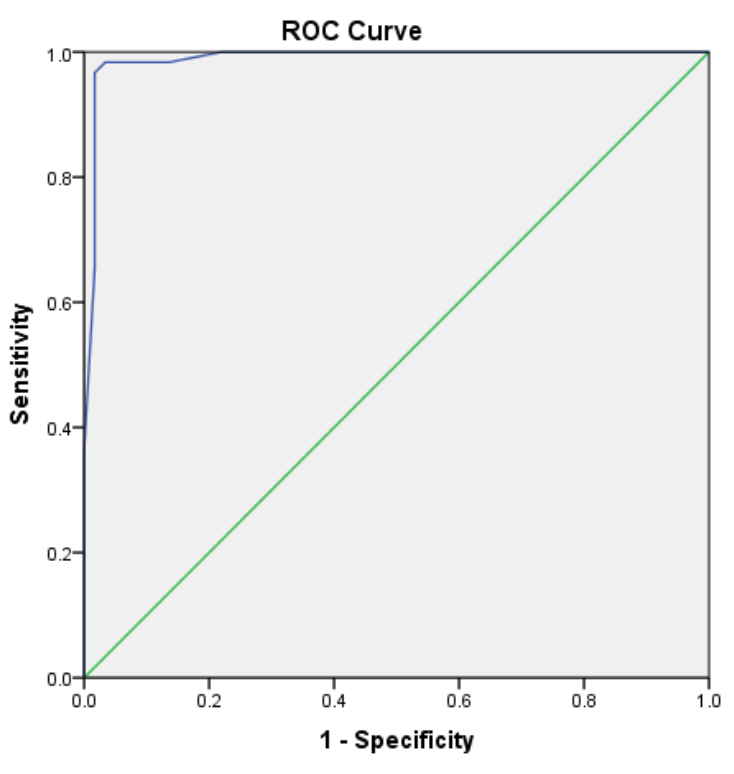

Diagonal segments are produced by ties.

Figure 1. Area Under the ROC Analysis to Determine the Value of DN4 for Predicting Neuropathic Pain (AUC=0.989, Sensitivity $=98.36$, Specificity $=98.31,95 \% \mathrm{Cl}=0.971-1.000)$.

with lower pain severity (AUC: 0.999 versus 0.988 ).

We also assessed the value of DN4 to diagnose mixed pains. Overall, 15 patients with mixed pain (8 women and 7 men, mean age 51 years) were assessed. The mean BMI in those patients was $25.86 \mathrm{~kg} / \mathrm{m}^{2}$, and the mean duration of pain was also 26.38 months. The Receiver operating characteristic (RTOC) analysis showed a high value for DN4 for discrimination of neuropathic pain group from non-neuropathic pain in the mixed subgroup (AUC: 0.977 ). The best cutoff point for DN4 to predict neuropathic pain in the mixed pain group was $\geq 5$, with a sensitivity of $88.9 \%$ and $100 \%$ specificity.

\section{Discussion}

According to our study, the Persian version of DN4 questionnaire was a valid and reliable tool for assessing neuropathic pain among our population. By modifying the structure of the original version of DN4 (for the first time) and achieving a new modified format (mainly by adding the VAS as a new arm), the modified version could more effectively differentiate non-neuropathic from neuropathic pain. In the study population, those suspected of having neuropathic pain, the modified DN4 questionnaire's assessment can effectively discriminate neuropathic pain from other non-neuropathic sources of pain.

We also highlighted the discriminative value of the modified DN4 concerning the baseline pain duration and severity, emphasizing the importance of adding some items related to the quality and duration of pain in the original format of DN4 as performed in our survey.

In a similar survey in the Iranian population, the sensitivity, specificity, and Youden Index in cutoff point $\geq$ 4 were $90 \%, 95 \%$, and 0.85 , respectively, with a Cronbach's alpha coefficient of 0.852 for the whole scale and the inter-rater agreement and test-retest reliability of 0.957 and 0.918 , respectively, ${ }^{10}$ which were very close to our observations. In a study by Chatila et al ${ }^{11}$ in 2017 using a new Arabic version of the DN4, internal consistency for the whole DN4questionnaire was 0.86 , and the intraclass correlation coefficient was also 0.99 . The test-retest reliability kappa coefficient for each item ranged from 0.92 to 1.00 . Furthermore, the AUCs of 0.97 and 0.94 were reported for the 10 -item DN4 and the 7 -item DN4, respectively.

A specificity of $82.3 \%$ and sensitivity of $97 \%$ were obtained for the 7-item DN4 at the threshold score of 3. On the other hand, the specificity of $95.8 \%$ and sensitivity of $93.0 \%$ were seen for the 10 -item DN4 at the cutoff point of 5 , which were similar to our study. It was first shown that the Arabic version of this tool is also valid for assessing neuropathic pain. Also, the best cutoff point for the DN4 scale can be similar to that obtained in our population. In a similar survey on the Greek version of DN4, ${ }^{12}$ the AUC was 0.92 . At the threshold of $>4$, a $93 \%$ sensitivity and a specificity of $78 \%$ were achieved for the DN4 scale for discerning non-neuropathic from neuropathic pain.

In a similar experience on the Spanish version of the $\mathrm{DN} 4{ }^{13}$ the questionnaire was reliable with the Cronbach's alpha coefficient of 0.71 , inter-rater agreement coefficient of 0.80 and test-retest intra-class correlation coefficient of that was very similar to our study. They also indicated its high validity with obtaining a cut-off value $>4$ points, which was the best value to discriminate

Table 3. Diagnostic Performance of DN4 at Different Cutoff Points

\begin{tabular}{lcccccc}
\hline Cut-off & Sensitivity & Specificity & $\begin{array}{c}\text { Positive Likelihood } \\
\text { Ratio }\end{array}$ & $\begin{array}{c}\text { Negative } \\
\text { Likelihood Ratio }\end{array}$ & $\begin{array}{c}\text { Positive Predictive } \\
\text { Value }\end{array}$ & $\begin{array}{c}\text { Negative } \\
\text { Predictive Value }\end{array}$ \\
\hline $3 \leq$ & 98.36 & 86.44 & 7.25 & 0.02 & 88.24 & 98.08 \\
$(\mathrm{Cl}: 91-99)$ & $(\mathrm{Cl}: 75-93)$ & $(\mathrm{Cl}: 75-93)$ & $(\mathrm{Cl}: 0-0.13)$ & $(\mathrm{Cl}: 79-93)$ & $(\mathrm{Cl}: 87-99)$ \\
$4 \leq$ & 98.36 & 96.6 & 29.02 & 0.02 & 96.77 & 98.28 \\
& $(\mathrm{Cl}: 91-99)$ & $(\mathrm{Cl}: 88-99)$ & $(\mathrm{Cl}: 7.4-113.3)$ & $(\mathrm{Cl}: 0-0.13)$ & $(\mathrm{Cl}: 88-99)$ & $(\mathrm{Cl}: 88-99)$ \\
$5 \leq$ & 98.36 & 98.31 & 58.03 & 0.02 & 98.36 & 98.31 \\
& $(\mathrm{Cl}: 91-99)$ & $(\mathrm{Cl}: 90-99)$ & $(\mathrm{Cl}: 8.5-405)$ & $(\mathrm{Cl}: 0-0.12)$ & $(\mathrm{Cl}: 89-99)$ & $(\mathrm{Cl}: 89-99)$ \\
$6 \leq$ & 96.72 & 98.31 & 57.07 & 0.03 & 98.33 & 96.67 \\
& $(\mathrm{Cl}: 88-99)$ & $(\mathrm{Cl}: 90-99)$ & $(\mathrm{Cl}: 8-398)$ & $(\mathrm{Cl}: 0.01-0.13)$ & $(\mathrm{Cl}: 89-99)$ & $(\mathrm{Cl}: 88-99)$ \\
\hline
\end{tabular}


neuropathic from non-neuropathic pains. In total, reviewing the literature shows that various versions of $\mathrm{DN} 4^{14}$ tested in different populations are reliable and valid to predict neuropathic pain. Also, the best cutoff value of DN4 to differentiate neuropathic from nonneuropathic pain is in the range of 4 to 5 , achieving acceptable sensitivity and specificity.

\section{Conclusion}

In our study, applying the new modified Persian version of DN4 achieved by adding a VAS score and a new question related to the appearance of pain in an especial joint led to high reliability and validity for this tool to discriminate neuropathic from non-neuropathic pain.

\section{Authors' contributions}

SPM: designing the study, reading and approving the final version of the manuscript; KM and SA: data collection; SE: data analysis. All authors equally contributed to writing the manuscript.

\section{Ethical Approval}

Declaration of Helsinki principles were respected. Entering the study was subject to the prior written consent of the patients. The principal researcher had adequately explained the objectives and methodology, and informed consent was signed before recruiting the patient.

\section{Competing Interests}

None to be declared.

\section{Acknowledgements}

This article is taken from Dr. Shahla Asadi's specialized doctoral dissertation number $61 \mathrm{M}$.

\section{References}

1. McCarberg B, D’Arcy Y, Parsons B, Sadosky A, Thorpe A, Behar R. Neuropathic pain: a narrative review of etiology, assessment, diagnosis, and treatment for primary care providers. Curr Med Res Opin. 2017;33(8):1361-1369. do i:10.1080/03007995.2017.1321532

2. Machelska H, Celik M. Recent advances in understanding neuropathic pain: glia, sex differences, and epigenetics. F1000Res. 2016;5:2743. doi:10.12688/f1000research.9621.1

3. Pratt $\mathrm{CH}$, King LE, Jr., Messenger AG, Christiano AM,
Sundberg JP. Alopecia areata. Nat Rev Dis Primers. 2017;3:17011. doi:10.1038/nrdp.2017.11

4. Zilliox LA. Neuropathic pain. Continuum (Minneap Minn). 2017;23(2):512-532. doi:10.1212/con.0000000000000462

5. Meacham K, Shepherd A, Mohapatra DP, Haroutounian S. Neuropathic pain: central vs. peripheral mechanisms. Curr Pain Headache Rep. 2017;21(6):28. doi:10.1007/s11916017-0629-5

6. Morgan KJ, Anghelescu DL. A review of adult and pediatric neuropathic pain assessment tools. Clin J Pain. 2017;33(9):844-852. doi:10.1097/ajp.0000000000000476

7. Deng Y, Luo L, Hu Y, Fang K, Liu J. Clinical practice guidelines for the management of neuropathic pain: a systematic review. BMC Anesthesiol. 2016;16:12. doi:10.1186/s12871-015-0150-5

8. VanDenKerkhof EG, Stitt L, Clark AJ, et al. Sensitivity of the DN4 in screening for neuropathic pain syndromes. Clin J Pain. 2018;34(1):30-36. doi:10.1097/ajp.0000000000000512

9. Canadian Agency for Drugs and Technologies in Health (CADTH). Diagnostic Methods for Neuropathic Pain: A Review of Diagnostic Accuracy. Ottawa, ON): CADTH; 2015.

10. Madani SP, Fateh HR, Forogh B, et al. Validity and reliability of the Persian (Farsi) version of the DN4 (Douleur Neuropathique 4 questions) questionnaire for differential diagnosis of neuropathic from non-neuropathic pains. Pain Pract. 2014;14(5):427-436. doi:10.1111/papr.12088

11. Chatila N, Pereira B, Maarrawi J, Dallel R. Validation of a new Arabic version of the neuropathic pain diagnostic questionnaire (DN4). Pain Pract. 2017;17(1):78-87. doi:10.1111/papr.12419

12. Sykioti P, Zis P, Vadalouca A, et al. Validation of the Greek version of the DN4 diagnostic questionnaire for neuropathic pain. Pain Pract. 2015;15(7):627-632. doi:10.1111/papr.12221

13. Perez C, Galvez R, Huelbes S, et al. Validity and reliability of the Spanish version of the DN4 (Douleur Neuropathique 4 questions) questionnaire for differential diagnosis of pain syndromes associated to a neuropathic or somatic component. Health Qual Life Outcomes. 2007;5:66. doi:10.1186/1477-7525-5-66

14. Bouhassira D, Attal N, Alchaar H, et al. Comparison of pain syndromes associated with nervous or somatic lesions and development of a new neuropathic pain diagnostic questionnaire (DN4). Pain. 2005;114(1-2):2936. doi:10.1016/j.pain.2004.12.010 